Cahiers de recherches médiévales

Journal of medieval studies

$4 \mid 1997$

Être père à la fin du Moyen Âge

\title{
Basin, un père aimant et tendre dans la chanson d'Auberi le Bourgoin
}

Isabelle Weill

\section{(2) OpenEdition \\ 12 Journals}

Édition électronique

URL : https://journals.openedition.org/crm/964

DOI : $10.4000 / \mathrm{crm} .964$

ISSN : 1955-2424

Éditeur

Honoré Champion

Édition imprimée

Date de publication : 15 décembre 1997

ISSN : 1272-9752

Référence électronique

Isabelle Weill, «Basin, un père aimant et tendre dans la chanson d'Auberi le Bourgoin », Cahiers de recherches médiévales [En ligne], 4 | 1997, mis en ligne le 15 janvier 2007, consulté le 15 décembre 2022. URL : http://journals.openedition.org/crm/964 ; DOI : https://doi.org/10.4000/crm.964

Ce document a été généré automatiquement le 15 décembre 2022.

Tous droits réservés 


\title{
Basin, un père aimant et tendre dans la chanson d'Auberi le Bourgoin
}

\author{
Isabelle Weill
}

Les chansons de geste sont connues pour mettre en scène des guerriers farouches dont les préoccupations parentales se réduisent au souci de la gloire et de la pérennité de leur lignage : dès que les jeunes atteignent l'âge adulte, les pères (Aymeri de Narbonne en est le représentant le plus célèbre) les obligent avec une extrème violence à quitter leurs terres afin qu'ils puissent par eux-mêmes faire l'épreuve de leur vaillance et se montrer dignes de leurs ancêtres. Quand on parle de jeunes enfants, il s'agit d'orphelins, de bébés enlevés par des bêtes féroces ou par des voleurs, d'enfants chassés avec leur mère soupçonnée injustement d'adultère, ou subsistant dans la profondeur des forêts avec un père malade ou ermite, voire d'enfants assassinés, toutes situations extrèmes excluant évidemment l'étude de la place que pouvait tenir le père auprès de ses fils et de ses filles. Mais dans ce qu'on appelle les chansons du deuxième âge épique, les narrateurs veulent renouveler le genre et ne répugnent plus à introduire des éléments dont le registre est extérieur à l'univers de la Geste et à s'intéresser à des héros qui ne sont plus entièrement conformes au canon épique. Notre étude portera sur un texte postérieur à 1250, la chanson d'Auberi le Bourgoin, conservée dans cinq manuscrits ${ }^{1}$, ce qui paraît la preuve d'un succès certain auprès du public ;le début illustre le thème carolingien traditionnel de l'adolescent contraint par une trahison ou par un scandale de s'exiler loin des siens mais la séparation entre le père nourricier et le fils de douze ans n'ayant pratiquement jamais connu sa mère est dépeinte avec un sentimentalisme tout à fait étonnant. Basin, un ancien féal de Charles Martel, qui a reçu en récompense pour ses services le duché de Dijon de Genève et de Vienne, voudrait finir paisiblement ses jours en se consacrant à l'éducation de son fils, mais sa deuxième épouse, liguée avec les perfides oncles de l'orphelin pour capter l'héritage, contraint le malheureux à confier l'enfant à son frère, provoque l'attaque du duché par le roi Desier de Pavie qui jette le duc vaincu au fond d'une profonde chartre dont il ne sortira jamais. Nous allons donc découvrir l'importance du rôle paternel, de façon positive à travers la tendresse et la prévenance dont fait preuve Basin, puis de façon négative à travers tous les actes de négligence volontaires auxquels se livre 
l'oncle félon qui avait pourtant juré sur des reliques de prendre soin du petit Auberi. Mais comment savoir si cette histoire de jeune adolescent élevé par des hommes peut correspondre aux réalités de la société aristocratique du XIII siècle ? On peut répondre en arguant que le narrateur fait par ailleurs preuve d'un étonnant réalisme psychologique: Auberi n'est pas un héros "lisse», ses mœurs, son caractère et sa conduite ne rappellent en rien l'austère rigidité d'un Roland, l'ardent dévouement à la cause chrétienne d'un Guillaume d'Orange, le souci du lignage d'un Garin le Lorrain ou d'un Gerbert de Mez. Au terme de ses "enfances", il se manifeste par une violence extrème en assasssinant en série ses cousins patrilinéaires et matrilinéaires (les psychologues modernes imputeraient cette agressivité au fait que le malheureux a été maltraité, battu et affamé en permanence depuis l'âge de douze ans par ses collatéraux désireux de faire discrètement disparaître l'héritier gênant);il devient un homme « léger ", c'est-à-dire irréfléchi, ne pensant nullement à venger son père et à reprendre la Bourgogne, acordant sa confiance au premier venu, s'effondrant face aux catastrophes et ne tirant aucune leçon des déconvenues successives ;il est assurément d'une vaillance à toute épreuve mais il se laisse presque corrompre par l'étalage des richesses et les paroles séduisantes du seigneur-bandit des Ardennes à l'attrait plus que trouble, le traître Lambert d'Oridon; il a fait un beau mariage en épousant Guibourg, la sœur de Pépin et la veuve du roi Orri de Bavière, mais, comme le lui fait remarquer perfidement Lambert, son épouse est une vielle femme ridée à qui les soins de beauté quotidiens ne peuvent rendre la jeunesse et son propre statut d'époux de la reine reste fort aléatoire. Le récit est certes situé à l'époque de Pépin le Bref mais on peut néanmoins y reconnaître bien des traits du XIII ${ }^{e}$ siècle : nous trouvons une description géographiquement exacte pour la France du Nord, nous voyons l'abbaye de Saint-Denis jouer un rôle important, la commune prendre les armes, les vassaux insoumis se réfugier dans leurs châteaux (les constructions fortifiées du XIII ${ }^{\mathrm{e}}$ siècle, constituées d'un palais et d'une tour) et en être délogés par les troupes royales. On peut donc affirmer qu'un texte faisant preuve d'un tel réalisme dans la peinture des caractères et de la société pourrait s'avérer également un témoin fidèle des mœurs du temps.

2 Au début du récit, le petit Auberi est âgé de douze ans et l'on penserait que son père le laisserait sans surveillance et jugerait que le préadolescent est responsable de ses actes ;c'est là notre première erreur. Nous sommes à la Saint Martin, donc à la minovembre, Basin revient de l'église et retourne au palais dans l'intention d'aller « en la riviere rivoier » c'est à dire d'aller chasser sur les bords des rivières et des étangs des oiseaux comme le héron avec l'aide de faucons; on s'attendrait à ce qu'il emmène son fils mais la narration nécessite une dispute entre Auberi et la marâtre qui cherche une occasion pour faire reconnaître à l'enfant ses propres droits sur les biens de son époux. Peut-être fait-il trop froid, peut-être l'enfant est-il trop jeune pour ce type de chasse, toujours est-il que Basin laisse son fils « esbanoier ", s'amuser sous la surveillance de sa femme et de son frère, mais ne manque pas en partant de leur adresser des recommandations concernant ce fils chéri, valables jusqu'à son retour :

«Quant dut errer, ses prent a aresnier:

«Veschi mon fils Auberi que j'ai chier,

Jel vous commant dusqu'a mon repairier. » « $\left(\mathrm{T}, \mathrm{f}^{\circ} 1 \mathrm{c}\right)$

3 Il se tourne ensuite vers l'enfant: »Au departir le corut embrachier.». Le manuscrit $\mathrm{H}$, qui insiste volontiers sur les éléments dramatiques, donne une variante : il «ala son fil baisier », ce qui montre l'importance attachée à la sensibilité dans ce texte. Ce n'est pas 
une notation isolée car Basin ne cesse en toutes circonstances de penser à son fils ;nous ne pouvons imaginer qu'il aurait pu faire partie des pères de la Geste mettant leur fils adulte à la porte, sa réaction devant son pays mis à feu et à sang par les envahisseurs lombards en est la preuve: dépourvu de troupes et d'alliés, il se livre d'abord au désespoir, pleure et arrache les poils de sa barbe, se lamente sur le fait d'être séparé de son fils puis imagine l'aide que l'enfant devenu adulte, en âge de ceindre une épée, aurait pu lui apporter. En quittant sa femme, pour laquelle il n'a pas un seul mot de tendresse, fait rarissime dans ce type de texte, il lui demande de lui faire envoyer des renforts et ses derniers mots sont des paroles instantes de recommandation pour l'enfant : je vous prie,si je meurs, au nom de Dieu qui souffrit le martyre pour nous, de faire élever mon fils jusqu'à ce qu'il puisse «la grande terre tenir »;la «male» Hermesent promet de le faire "esgarder et nourrir » mais se jure bien de faire mourir le père et le fils. Le manuscrit $\mathrm{H}$ fait prononcer à Basin quatre séries de lamentations supplémentaires sur l'orphelin sans mère qui va perdre aussi son père ;le souvenir du fils l'emporte même sur les prières du guerrier chrétien attaqué de toutes parts et voyant sa fin prochaine :

« Dex, dist Basin, sainte Virge onoree,

Recevés m'ame, que ma vie est alee!

Auberi fix, com dure destinee! « $\left(10 \mathrm{v}^{\circ}\right)$

Avant de quitter définitivement la scène, Basin, jeté dans une profonde chartre à Pavie, ne se préoccupe que de son fils, promis, pense-t-il, à une mort certaine :

Or fu Basin en la chartre avalé

Et nuit et jour s'est forment desmentés :

« Auberi fius, jamais ne me verrés,

En prison sui, tu ieres mal gardés

Plus sui de vos coureciés et irés

Que de mon mal dont je ai trop asés.

Bien sai, biau fius, qu'a mort estes livrés.

Tu n'ieres pas servis ne hounourés,

Ains t'ocira Henris li desfaé

Car d'Ermesent en a esté loués.

Quant il sauront qu'iere enprisounné,

Auberis fius, tost serés afolés! $(\mathrm{R}, 1)$

On peut penser que cet amour paternel poussé au paroxysme est décrit avec insistance (selon une technique qui annonce le roman feuilleton et les séries télévisées modernes) afin de dramatiser, d'une part, les souffrances d'un loyal guerrier qui voit s'effondrer tous ses repères familiaux et vassaliques et, $d$ 'autre part, les peines qui vont accabler Auberi, thème principal de la narration;mais la chanson de geste connaît d'autres exemples de cet amour dénué d'égoïsme: dans Daurel et Beton ${ }^{2}$, un texte occitan du $\mathrm{XII}^{\mathrm{e}}$ siècle rattaché à la chanson française de Beuve de Hantone, le roi Beuve est traîtreusement blessé à mort au cours d'une chasse au sanglier par son ami et héritier Gui qui convoite sa femme et son royaume; avant de mourir, le roi explique à son meurtrier comment maquiller sa mort en accident de chasse, mansuétude peu commune qui trouve rapidement une explication: le mourant tente de négocier avec son meurtrier la vie sauve pour son fils, le petit Beton, âgé d'un an qui n'aurait droit qu'à la moitié de l'héritage, toutes choses que refuse le traitre qui jure de tuer l'enfant et de s'approprier tout le royaume;seul le dévouement du jongleur Daurel pourra finalement sauver l'enfant. Il ne faudrait pas sur ce point oublier de comparer ces textes avec un texte bien plus ancien et bien plus connu, La Chanson de Roland, texte 
fondateur, en quelque sorte, de la tradition épique : Ganelon a été désigné pour servir de messager auprès de Marsile, rôle dangereux entre tous étant donné le sort qui a été réservé aux messagers précédents et qu'on n'aurait pas dû confier à un homme aussi proche du roi qu'est son beau-frère, selon l'intéressé :

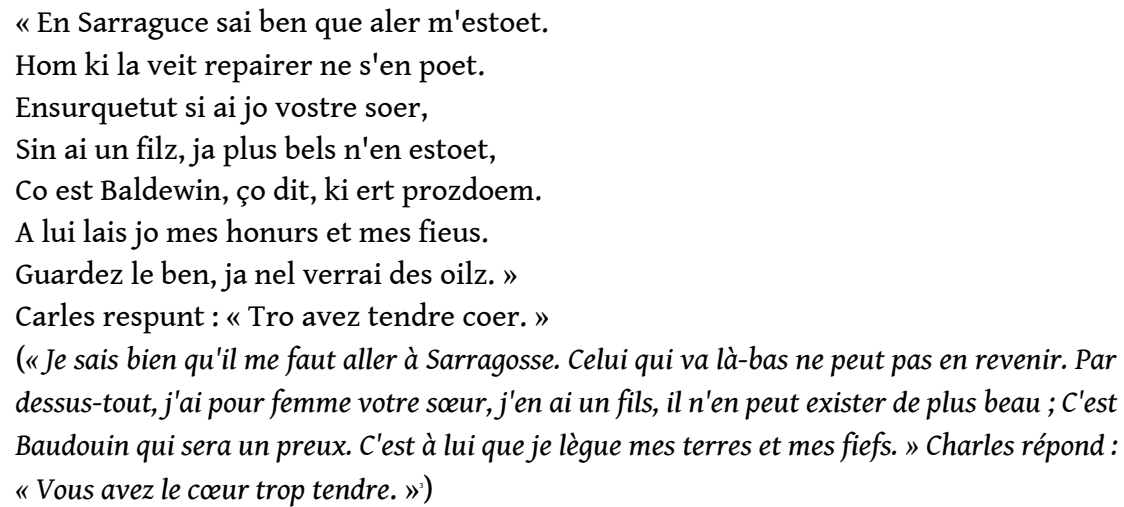

6 Notons que nous trouvons, dans ce texte du XI ${ }^{\mathrm{e}}$ siècle, l'image du père attendue, celle d'un homme qui ne voit en ce fils que le digne continuateur de son lignage ;cette manifestation de tendresse, pourtant bien contenue, paraît déplacée chez un guerrier accomplissant un service et n'éveille que des sarcasmes de la part du roi

7 Administrer avec sévérité punitions et corrections fait partie du rôle que l'on dévoluerait traditionnellement au père mais on ne trouve pas dans l'épopée des exemples de jeunes enfants punis ou fouettés: dans la chanson du XIV ${ }^{\mathrm{e}}$ siècle de Baudoin de Sebourc ${ }^{4}$, le traître Gaufroi épouse Rose de Beauvais après avoir tué son mari; le jour des noces, le plus jeune des orphelins de Beauvais, Bauduin, âgé de trois ans environ, insulte son beau père, lequel exige une punition exemplaire, ce qui scandalise tout le monde et ce qui est évidemment refusé par la mère.Revenons au début de notre texte et retrouvons Basin à son retour de la chasse. À peine est-il descendu de cheval que son épouse vient se plaindre de l'enfant qui, dit-elle, menace de la faire chasser du domaine et de la tuer (l'enfant a de fait refusé de lui reconnaître le moindre droit sur l'héritage qu'elle revendiquait comme son douaire); elle déclare ne plus jamais vouloir entrer dans le lit de Basin si on ne lui rend pas immédiatement justice ;littérairement les hommes cèdent toujours à ce type de menace ${ }^{5}$ mais Basin n'a rien d'un homme faible: non seulement il n'envisage pas une seule seconde de punir l'enfant mais il défend à sa femme de le gronder parce qu'il a l'excuse de la jeunesse :

«Dame, dist-il, lessiez l'enffant ester :

Ja est il jones, nel devés ramporner

Et le l'aim molt, je nel quier a celer.

Se plus de moi puet Auberis durer,

Tote ma terre avra a governer. » (T 2a)

8 Ce refus catégorique de corriger les enfants se retrouve dans la deuxième partie de la chanson où nous verrrons évoqués les rapports d'un père et d'une fille, ce qui est rarissime dans ce type de texte : il s'est écoulé une dizaine d'années depuis le début des événements et Auberi, devenu l'époux de la reine de Bavière, reçoit la visite d'un voisin, Lambert d'Oridon (en fait ce traître convoite la main et l'héritage de Seneheut, l'unique fille du feu roi Orri de Bavière, belle-fille d'Auberi et fiancée à son fidèle neveu Gasselin) qui se prétend son parent et qui l'éblouit par un étalage incroyable de cadeaux de prix. Le nouvel arrivant émet la prétention de d'embrasser la jeune fille et de la combler de présents en échange de ses baisers, ce qu'elle refuse avec la hauteur et 
l'indignation que l'on devine; Auberi, furieux de voir insulter son si généreux hôte, gratifie la malheureuse d'un bon coup de poing sur le nez, si violent qu'elle se retrouve couverte de sang. La reine Guibourg, avant de sortir soigner et consoler sa fille éplorée, admoneste son époux qui a outrepassé ses droits: il peut certes réprimander et conseiller sa "fillâtre » mais il doit savoir que jamais son père, le roi Orri, ne s'est livré sur elle à de pareils actes de violence :
« Auberi sire, or vos doi blastengier!
Moult avés fait Seneheut laidengier
Por cel vassal qu'el ne veut acointier.
Je ne di mie nel doiés enseignier :
Devant tous homes la devés chastoier.
Bien la poés ferir et laidengier,
Qu'el n'a ami qui or li vaigne aidier ;
En li mal faire poés vos bien pechier.
Se ele est orfe, Dieus la puist conseillier!
Onques ses peres qui fu rois de Baivier
Ne l'en fist tant, moult l'en doit anoier... "

La violence d'Auberi est condamnée par une épouse habituellement fort soumise, incapable d'offenser son mari devant un étranger sans raison grave;on peut en conclure qu'un père et a fortiori un parâtre n'ont pas du tout sur leur enfant les droits du paterfamilias antique.

Le refus de punir Auberi et surtout la reconnaissance publique de l'enfant en tant qu'héritier putatif de la Bourgogne (ne parlons pas des protestations d'amour paternel habituelles) remplissent Hermesent de fureur et la dame menace alors de se suicider :

«Basin, dist elle, me veus tu vergonder!

Se Auberi ne me vels delivrer

Ja me verras d'un coutel acorer. » (T 2b)

11 La menace en public émeut Basin qui se voit contraint de devoir se séparer de son fils pour assurer sa sécurité et l'idée de ne pouvoir veiller lui-même sur son enfant le met hors de lui :

« Deus, dist Basin, com or puis forsener

Quant je mon fil ne puis o moi tenser.»

Le verbe «tencier ",qui appartient plutôt au vocabulaire juridique, implique l'idée de défendre, d'apporter secours et aide et nous retrouvons là l'image traditionnelle du père protecteur. On voit que Basin n'a pas du tout le désir d'envoyer l'enfant terminer son éducation et commencer son apprentissage de chevalier loin de lui, auprès de son oncle maternel ou de son seigneur lige qui serait en l'occurrence le roi Pépin. Henri d'Autun, le frère du duc, corrompu par sa belle-sœur, propose alors qu'on lui confie l'enfant jusqu'à ce qu'il soit en âge de "ses garnemens porter", de supporter le poids des armes. Les Bourguignons qui ignorent tout du complot ourdi par Hermesent et Henri conseillent à Basin de confier l'enfant à son oncle et Basin ne peut que céder devant la volonté générale qui vient de s'exprimer; il prend toutefois la précaution de faire jurer à son frère sur des reliques qu'il assumera sa mission de substitut paternel avec toute la fidélité possible (la suite du texte prouve qu'il a dû exiger de lui non pas un serment général mais des paroles précises concernant chaque acte de la vie courante). Henri a un statut très inférieur à Basin, on lui confie l'enfant comme on le confierait à un serviteur fidèle . Basin aurait pu envisager une autre possibilité : par sa mère Erembourg, morte au bout de sept ans de mariage, Auberi a un oncle maternel, le 
puissant Oedes de Langres (un personnage du Cycle Carolingien) et en toute logique textuelle, c'est auprès de lui que Basin devrait envoyer l'enfant ; seulement Oedes, à la suite du complot contre Charles Martel déjoué avec l'aide du «larron» Basin, a perdu son duché qui a été attribué à son beau-frère (toutes ses tentatives pour le récupérer ont échoué face à la détermination de Basin) et on comprend que Basin ne puisse avoir de recours de ce côté.

La séparation du père et du fils sera définitive : laissant Basin au fond de sa prison, la chanson s'attache désormais à décrire le sort d'Auberi «nourri» loin de son père chéri ;il est rarissime de voir l'éducation d'un enfant décrite et nous allons la trouver inscrite "en négatif » dans une sorte de litanie : Henri «s'est mal prouvé », n'a pas tenu parole et n'a pas répondu à l'attente de Basin et l'absence du père va rejaillir sur les soins d'hygiène, la nourriture,l'éducation, les marques d'affection et de respect, les cadeaux dus à l'héritier de la Bourgogne. L'enfant, dit le texte, n'est jamais ni peigné ni lavé, ses "draps ", c'est à dire ses vêtements sont déchirés, "desrous ", en lambeaux et même, comme le précise le seul manuscrit $\mathrm{H}$, « despanés », c'est à dire ayant perdu leur chaude doublure matelassée et piquée. Son lit n'est jamais «fait ni paré», c'est à dire qu'il est réduit à une paillasse, sans oreiller, sans drap et sans couverture ;dans la suite du texte on voit l'adolescent "monter le planchier» et se coucher seul avec deux lévriers qui le réchauffent la nuit, un par devant et un par derrière. On ne s'attend évidemment pas à voir un père de milieu aristocratique laver son enfant et lui faire son lit mais le texte implique qu'un père digne de ce nom ou son substitut aurait veillé à ce que toutes ses tâches soient accomplies et n'aurait pas toléré que l'enfant soit laissé en guenilles dans la crasse devant lui. Henri aurait dû donner les ordres voulus puisqu'il

devoit le faire honourer et servir

Et conreer et chaucier et vestir. (R 2)

Le pauvre Auberi est moins bien traité qu'un esclave vendu aux Turcs ;plus grave encore il est privé de sommeil par ses cousins plus âgés qui le réveillent aux aurores pour le tyranniser, et, qui pis est, il est volontairement sous-alimenté : quand on passe à table, Henri, au lieu de faire asseoir son neveu à côté de lui à la première place le fait chasser et le relègue au dernier bout là où les cuisiniers ne passent aucune nourriture. Le manuscrit $\mathrm{H}$ en rajoute encore (" sovent estoit avec les chiens trovés») en lui faisant partager la pâtée des chiens. Auberi doit son salut aux chevaliers, les anciens amis de son père, qui lui jettent discrètement, les uns un morceau de pain, les autres un quartier et même un pain entier. Ces mauvais traitements n'empêchent nullement Auberi de devenir un jeune homme "menbru ", c'est à dire athlétique...

L'éducation qu'un adolescent doit recevoir de son père est également inscrite dans le texte : un père doit donner à son fils le goût des armes et des chevaux et doit donc lui en procurer, il doit l'emmener chasser en forêt, c'est à dire tirer à l'arc sur le gibier à poil, et en "riviere ", c'est à dire lui apprendre à lancer ses faucons mués (es oiseaux ayant fait plusieurs mues sont plus solides et ont plus de valeur) sur les canards, les grues, les hérons au bord des étangs et des rivières :

Hensis ses oncles s'est vers lui parjurés

Qui lui jura seur tous sains honourés

Que il seroit de tous biens aiaisiés :

Chevax et armes li donroit il assés

Et le menroit chacier el bois ramés 
Et en rivière o lé faucons mués.

Del tot en tot s'est vers lui parjurés. (R 2)

On peut constater qu'aucune éducation intellectuelle n'est prévue, ce qui prouve bien que nous n'avons dans ce passage que ce qui était expressément à la charge du père. Auberi ne souffre pas trop de ces carences car il est non seulement d'une santé à toute épreuve mais encore assez bien doué pour se faire lui-même sa propre éducation : il apprend tout seul à se servir de l'équipement du chevalier en regardant les autres et s'exerce à l'escrime sur des "tallevas", des boucliers et...sur les jeunes gens du domaine, les «donziaux du régné». Le texte précise qu'il arrive à un résultat plus qu'honorable, quelque dépit que puisse en éprouver son "parage», c'est à dire ses cousins et oncle.

Personne ne le traite avec respect (les inférieurs imitent la conduite de leur maître) ni même avec politesse ou gentillesse, il n'est jamais «bel apelés» et il est battu en permanence par les deux fils de Henri, ses cousins qui doivent être plus âgés que lui (Basin n'a pu se marier que fort tard, il a une fille bâtarde, marièe et mère de fils de l'âge d'Auberi); les deux tourmenteurs réveillent leur jeune cousin dès l'aube pour l'obliger à faire du saut en longueur sur le «fumier» (le tas de compost et d'ordures) ;Auberi se décide à porter plainte devant son oncle le jour où les méchants ont dissimulé dans le fumier des couteaux, la pointe de la lame en l'air :

«Par Dieu, sire oncles, molt me faites laidir.

Ja vos vi je a mon pere plevir

Que me feroies honorer et servir

Tant que porroies ma terre garantir.

Por coi me laises ne batre ne ferir?

Tu fais pechié, si t'en puet meschaïr!

Hé Basin pere, Dex vos puist beneïr

Et il vos lait de la prison eissir,

Encore vos puisse et baisier et joïr! » (H 1')

18 Henri, furieux devant ce rappel de l'amour qui unit l'enfant au frère haï et jalousé, ordonne à ses fils devant Auberi de redoubler de coups envers leur cousin. Le sort en est alors jeté, l'éducation à rebours est terminée et Auberi, pour échapper à une mort certaine, va s'enfuir de chez son oncle mais non sans avoir accompli sa vengeance; il se fait forger une guisarme (une arme terrible avec une longue lame et un long manche) qu'il paye avec du pain qu'il avait mis de côté, il la dissimule dans le fumier et assassine ses cousins avant qu'ils ne l'assassinent lui-même. Cette violente transgression répond à la trahison d'Henri, qui avait juré d'être un père pour Auberi et qui retrouve ses enfants morts sur le fumier ;il faut noter qu'Henri n'est pas Basin et qu'il pense beaucoup plus à tuer son neveu et à accomplir le serment qu'il avait fait à la marâtre qu'à pleurer la perte qu'il vient de subir.

19 Que pouvons-nous conclure de la représentativité d'un texte littéraire de cet ordre pour reconstituer l'image du père dans la société médiévale ? Il est certain que ce motif $\mathrm{du}$ père aimant et tendre capable de remplacer la mère disparue dans son rôle traditionnel de nourricière n'est pas courant et il est sûr qu'il est narratologiquement utilisé pour mettre en valeur le thème principal des malheurs et des peines qui assaillent injustement Auberi, le héros de la chanson. Mais on peut se dire que trouver ce type de rapport dans ce genre hautement normé qu'est la chanson de geste -un genre qui se doit de transmettre l'image de l'homme viril par excellence, le guerrier, telle qu'on voudrait qu'elle soit et non pas telle qu'elle est -n'est pas dénué de 
signification; en effet, dans la chanson d'Auberi le Bourgoin, les transgressions multiples commises envers la thématique épique (tentation de la chair, du pouvoir et des richesses ;trahisons lignagères; refus d'obéir aux contraintes morales et sociales qui commandent de tout faire pour venger son père) sont expressément stigmatisées et par le narrateur et par les personnages du récit. Personne ne reproche jamais au guerrier que reste Basin jusqu'à sa mort (le vieillard prisonnier après un combat acharné est un motif épique ${ }^{7}$ ) d'avoir un cœur trop faible ou trop tendre et de faire preuve de faiblesse dans ses rapports avec son fils. L'idée «moderne» qu'un homme «maternant» ses enfants perd ses prérogatives et ternit son image virile est totalement étrangère à notre texte, quoiqu'il doive en coûter à l'histoire des mentalités.

\section{NOTES}

1. Il s'agit de $R$, Rome, Bibl. Apost. Vatic. Regina lat. $1441 ; H$, Berlin, Staadtsbibliothek Gall $4^{\circ} 48$; B, Paris, B.N. fonds fr. 860 ; C, Paris, B.N. fonds fr. 860 ; T, Paris, B.N. fonds fr. 24368. Le texte ( 35000 vers environ) n'a été édité que partiellement; on peut consulter pour le début P. Tarbé, Le Roman d' Aubery le Bourgoin, Reims, 1849, XXXII-106 p. et A. von Keller, Romwart, Beitraege zur Kunde Mittelalterlicher Dichtung aus itliaenischen Bibliotheken, Mannheim 1844 ( (CHR 1441 Auberis li Borgignons », pp.202-43).

2. Ed P. Meyer, Daurel et Beton, Paris, 1880 (SATF 13).

3. Édition et traduction de G. Moignet, La Chanson de Roland, Bordas, Paris, 1969, 320p., vv. 310-17.

4. Éd L. Boca, Li Romans de Bauduins de Sebourg, 2 vol., Valenciennes, 1841.

5. On peut penser à la façon dont la duchesse de Bourgogne arrive à convaincre son mari de lui révéler son secret dans La Châtelaine de Vergy (éd. J. Dufournet et L. Dulac, Folio, Gallimard, 1994), vv. 551-658.

6. I. Weill, Prolégomènes à une édition critique d'Auberi le Bourgoin, thèse de troisième cycle dactylographiée, Paris, 1986, 3 vol., CXXXII-569 p., p.35, R, vv. 289-98.

7.On peut se reporter au début de Gaufrey (éd. F. Guessard et P. Chabaille, VIieweg, Paris, 1849, LXVIII-332p.).

\section{AUTEUR}

\section{ISABELLE WEILL}

Université Paris X-Nanterre 